\title{
Gamma-ray and X-ray luminosities from spin-powered pulsars in the full polar cap cascade model
}

\author{
Bing Zhang and Alice K. Harding \\ Laboratory of High Energy Astrophysics, NASA Goddard Space Flight Center.
}

\begin{abstract}
We modify the conventional curvature radiation (inverse Compton scattering) + synchrotron radiation polar cap cascade model by including the inverse Compton scattering of the higher generation pairs. Within the framework of the space-chargelimited-flow acceleration model with frame-dragging proposed by Harding \& Muslimov (1998), such a full polar cap cascade scenario can well reproduce the $L_{\gamma} \propto\left(L_{\mathrm{sd}}\right)^{1 / 2}$ and the $L_{x} \sim 10^{-3} L_{\text {sd }}$ dependences observed from the known spin-powered pulsars. According to this model, the "pulsed" soft ROSAT-band X-rays from most of the millisecond pulsars might be of thermal origin, if there are no strong multipole magnetic components near their surfaces.
\end{abstract}

\section{INTRODUCTION}

Eight and 35 spin-powered pulsars have been also detected in $\gamma$-ray and Xray bands, respectively. Despite their great diversity of emission features, the luminosities of these pulsars seem to obey the empirical laws $L_{\gamma} \propto\left(L_{\mathrm{sd}}\right)^{1 / 2} \propto B / P^{2}$ (Thompson et al. 1997), and $L_{x} \sim 10^{-3} L_{\text {sd }} \propto B^{2} / P^{4}$ (Becker \& Trümper 1997), where $L_{\mathrm{sd}}$ is the spin-down luminosity of the pulsar. The spectra of the $\gamma$-ray emission and the X-ray emission from most of the pulsars are non-thermal, while full surface thermal emission components are identified from Vela, Geminga, PSR 1055-52 and PSR 0656+14, and possible hot polar cap thermal emission components are reported from PSR 1929+10 and PSR J0437-4715.

Two competing models for pulsar high energy emission, i.e., the polar cap models (Daugherty \& Harding 1996; Sturner, Dermer \& Michel 1995) and the outer gap models (Cheng, Ho \& Ruderman 1986; Zhang \& Cheng 1997) were proposed. Canonical polar cap cascade models involve the curvature radiation (CR) or inverse Compton scattering (ICS) of the primary particles and the synchrotron radiation (SR) of higher generation pairs. Here we modify such a cascade picture by including the ICS of the higher generation pairs, which is important since it usually occurs in the resonant regime. A more detailed presentation of this study is shown in Zhang \& Harding (1999). 


\section{THE MODEL}

\section{The "full-cascade" picture}

The "full-cascade" scenario is: primary particles accelerated from the inner gap emit primary $\gamma$-rays via CR or ICS, these $\gamma$-rays will pair produce in strong magnetic fields. The secondary pairs have non-zero pitch angle with respect to the field lines. The perpendicular energy of the pairs will go to high energy radiation via SR, and the parallel energy of the pairs will also convert to radiation via ICS with the soft thermal photons from either the full neutron star surface or the hot polar cap. Under the condition of $\gamma \geq \gamma_{\mathrm{res}}=48 B_{12} \operatorname{Max}\left[\left(1-\beta \mu_{\mathrm{s}, \max }\right) T_{s, 6},\left(1-\beta \mu_{\mathrm{h}, \max }\right) T_{h, 6}\right]^{-1}$ (i.e. the "resonant scattering condition", Dermer 1990), the efficiency of converting particles' kinetic energy to radiation by ICS is almost $100 \%$, so that the total high energy emission luminosity is approximately the polar cap particle luminosity. Here $T$ is the temperature of the soft photons, and $\mu_{\max }$ is the cosine of the maximum scattering angle. Note that two components, i.e., a soft full surface thermal emission (denoted by 's') and a hard hot polar cap thermal emission (denoted by ' $h$ '), are adopted.

The basic ingredients in constructing an analytic description of such a full cascade scenario are (1) the energy distribution in SR (perpendicular) and ICS (parallel) branches, and (2) the recursion relations between different generations. For the former, $\eta_{\|}=\gamma_{i, \|} / \gamma_{i}=\left[1+\left(\gamma_{i+1}^{2}-1\right) \sin ^{2} \theta_{\mathrm{kB}}\right]^{-1 / 2}$ and $\eta_{\perp}=1-\eta_{\|}$are the energy portions for the parallel (ICS) and the perpendicular (SR) branches, respectively, where $\gamma_{i}$ and $\gamma_{i, \|}$ are the total and parallel Lorentz factors of the $i$-th generation pairs, and $\theta_{\mathrm{kB}}$ is the impact angle between the photon and the magnetic field line. With this, one can get the reduction factor of the typical photon energies for the adjacent generations, e.g., $\kappa_{\mathrm{SR}}=\epsilon_{i+1, \mathrm{SR}} / \epsilon_{i}=(3 / 4) \chi$, and $\kappa_{\mathrm{ICS}}=\epsilon_{i+1, \mathrm{ICS}} / \epsilon_{i}=\eta_{\|} B_{e}^{\prime}$, where $B_{e}^{\prime}=B_{e} / B_{c r i}$, and $\chi$ is the parameter to describe the $\gamma-B$ pair production process. The photon escaping energy is $E_{\gamma, \text { esc }}(\mathrm{nPSR}) \simeq 2.0 \mathrm{GeV} B_{e, 12}^{-1} P^{1 / 2} r_{e, 6}^{-1 / 2} \chi_{1 / 16}$ for normal pulsars, and $E_{\gamma \text {,esc }}(\mathrm{msPSR}) \simeq 73 \mathrm{GeV} B_{e, 9}^{-1} P_{-3}^{1 / 2} r_{e, 6}^{-1 / 2} \chi_{1 / 12}$ for millisecond pulsars, where $\chi_{1 / 16}=\chi /(1 / 16)$, and $\chi_{1 / 12}=\chi /(1 / 12)$, and $r_{e, 6}$ is the emission height in units of $10^{6} \mathrm{~cm}$. Given the typical primary photon energy $E_{0}$ (which is model-dependent), we can then get some non-integer generation order parameters (Lu et al. 1994; Wei et al. 1997), e.g. $\zeta_{\mathrm{SR}}=\frac{\log \left(E_{\mathrm{esc}} / E_{0}\right)}{\log \left(\kappa_{\mathrm{SR}}\right)}+1$ (for pure SR generations), and $\zeta_{\mathrm{ICS}}=\frac{\log \left(E_{\text {esc }} / E_{0}\right)}{\log \left(\kappa_{\mathrm{ICS}}\right)}+1$ (for pure ICS generations), which can describe the complex cascade process analytically.

\section{Harding \& Muslimov acceleration model}

Harding \& Muslimov (1998) has improved the space-charge-limited flow acceleration model (Arons \& Scharlemann 1979) by incorporating the frame-dragging $E_{\|}$, upper and lower pair formation front and both the CR and ICS of the primary 
electrons. It was found that a stable accelerator is located at an effective "radius" of $R_{\mathrm{E}} \sim(1.5-2) R$ for normal pulsars when ICS energy loss is less than that of $\mathrm{CR}$, since the ICS of the upward versus downward particles with the soft thermal photons are anisotropic due to different geometries. The typical length of the CR-controlled gap is $S_{c} \simeq 4.8 \times 10^{4} \mathrm{~cm} B_{p, 12}^{-4 / 7} P^{4 / 7} R_{E, 6}^{16 / 7}(\cos \alpha)^{-3 / 7}$, and the typical Lorentz factor of the primary particles is $\gamma_{0}=4.7 \times 10^{7} B_{p, 12}^{-1 / 7} P^{1 / 7} r_{e, 6}^{4 / 7}(\cos \alpha)^{1 / 7}$, where $\alpha$ denotes the inclination angle of the neutron star. Thus the typical energy of the primary photons is $E_{0} \simeq 33.2(\mathrm{GeV}) B_{p, 12}^{-3 / 7} P^{-1 / 14} r_{e, 6}^{17 / 14}(\cos \alpha)^{3 / 7}$, with which one can get explicit expressions for the generation parameters, and complete the analytic description of the full-cascade model.

\section{LUMINOSITY PREDICTIONS}

\section{Gamma-ray luminosity}

An interesting feature of the Harding \& Muslimov model is that $\gamma_{0}$ is insensitive to pulsar parameters, so that the polar cap luminosity $L_{\mathrm{pc}}=\gamma_{0} m c^{2} \dot{N}_{p}$ is roughly proportional to $\left(L_{\mathrm{sd}}\right)^{1 / 2}$. One advantage of the full cascade model is that the ICS branches can convert the "lost" parallel kinetic energies of the particles also to radiation, so that the total high energy luminosity (mainly $\gamma$-ray luminosity) is also roughly proportional to $\left(L_{\mathrm{sd}}\right)^{1 / 2}$. More specifically, the model predicts

$$
\begin{aligned}
L_{\gamma}(\text { full }) \simeq L_{\mathrm{pc}} & =5.4 \times 10^{31} \mathrm{erg} \cdot \mathrm{s}^{-1} B_{p, 12}^{6 / 7} P^{-13 / 7} r_{e, 6}^{4 / 7}(\cos \alpha)^{8 / 7} \\
& =1.7 \times 10^{16} B_{p, 12}^{-1 / 7} P^{1 / 7} r_{e, 6}^{4 / 7}(\cos \alpha)^{8 / 7}\left(L_{\mathrm{sd}}\right)^{1 / 2} .
\end{aligned}
$$

This nearly reproduces the observed $L_{\gamma} \propto\left(L_{\mathrm{sd}}\right)^{1 / 2}$ feature.

\section{Thermal X-ray luminosity}

There are two thermal components in a pulsar's X-ray spectrum (though they might be buried under the non-thermal component). For the full surface thermal component, we have adopted a simple rough "standard" cooling model, which is not inconsistent with the observations.

For the hot polar cap thermal component, we treated it with the self-consistent polar cap heating in the Harding \& Muslimov model. Since the flow is space-chargelimited so that the deviation of local charge density $(\rho)$ from the Goldreich-Julian density $\left(\rho_{\mathrm{GJ}}\right)$ is small, the backflow particle luminosity should be only a small portion (a factor of $\left|\rho-\rho_{\mathrm{GJ}}\right| / 2 \rho_{\mathrm{GJ}}$ ) of the polar cap luminosity, which reads

$$
L_{x, t h, \max } \simeq 5.9 \times 10^{29} \mathrm{erg} \cdot \mathrm{s}^{-1} B_{p, 12}^{2 / 7} P^{-9 / 7} r_{e, 6}^{-22 / 7}(\cos \alpha)^{5 / 7},
$$

and the maximum polar cap temperature (assuming an area of $\pi r_{p}^{2}$, where $r_{p}=$ $\left.\theta_{\mathrm{pc}} R=1.45 \times 10^{4} P^{-1 / 2} \mathrm{~cm}\right)$ is 

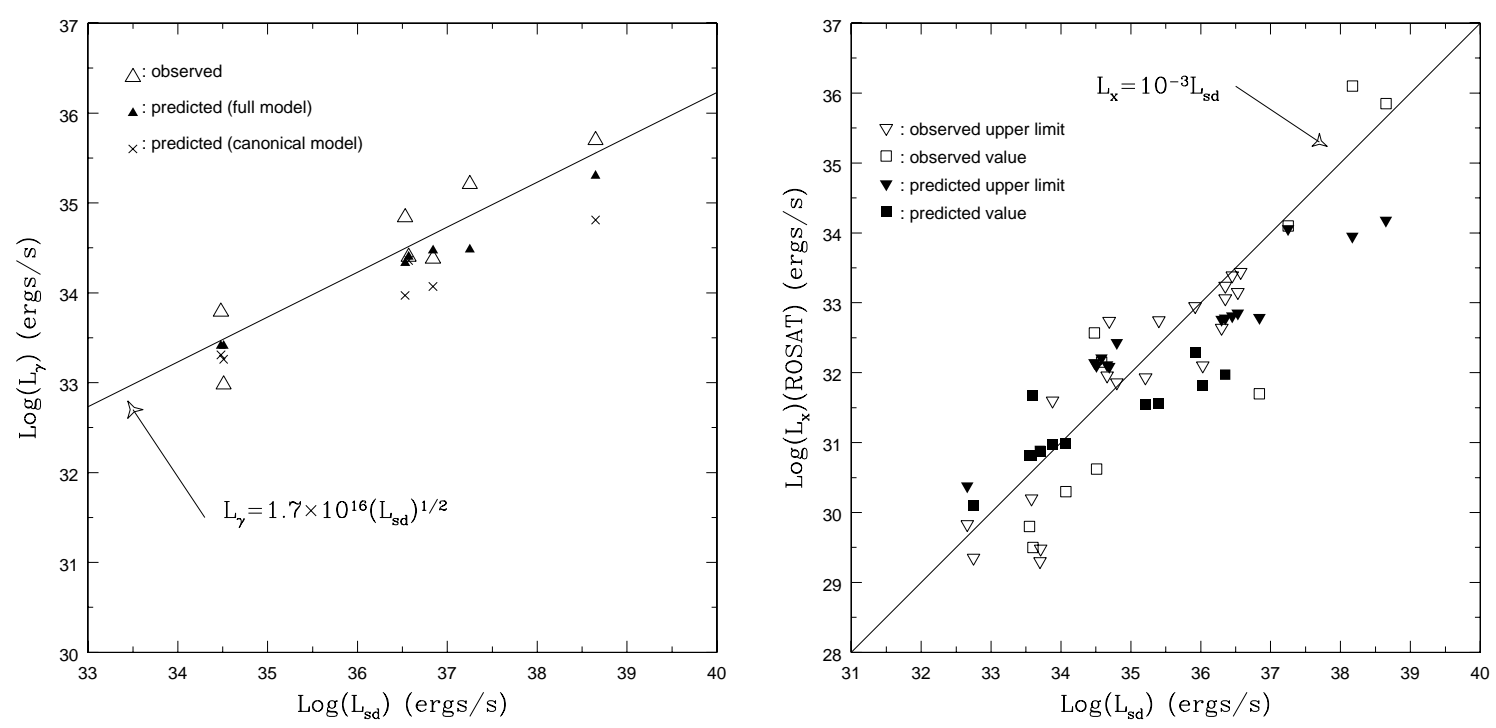

FIGURE 1. Observation versus theory: (a) $\gamma$-ray luminosities (b) X-ray luminosities.

$$
T_{\mathrm{pc}, \max }=2.0 \times 10^{6} \mathrm{~K} B_{p, 12}^{1 / 14} P^{-1 / 14} r_{e, 6}^{-11 / 14}(\cos \alpha)^{5 / 28}
$$

\section{Non-thermal X-ray luminosity}

The SR spectra of all SR branches can not get down to X-ray energies as those observed by ROSAT and ASCA, since there is a low energy cut-off at the blueshifted local resonant frequency (corresponding to the transition between the ground and the first excited Landau levels). Another advantage of the full cascade scenario is that, the ICS branches, which were neglected in the canonical cascade model, can naturally give a non-thermal component extend to the X-ray band. For normal pulsars, the maximum non-thermal luminosity below a certain energy $E_{c}$ could be estimated as

$$
L_{x, n t h}\left(E_{c}\right) \leq L_{\mathrm{pc}} \sum_{k=1}^{\operatorname{int}\left(\zeta_{\mathrm{SR}}\right)}\left[\eta_{\perp}^{k-1}\left(\sum_{j=1}^{\operatorname{int}\left(\zeta_{\mathrm{ICS}, \mathrm{k}-1}\right)} \eta_{\|}^{j} \eta_{c, k, j}\right)\right]
$$

where $\zeta_{\mathrm{ICS}, \mathrm{k}}=\frac{\log \left(E_{\mathrm{esc}} / E_{k}\right)}{\log \left(\kappa_{\mathrm{ICS}}\right)}+1$ is the number of pure ICS generations for the typical energy of the $k$-th SR generation, $E_{k}=E_{0} \kappa_{\mathrm{SR}}^{k}$, to reduce to the escaping energy $E_{\text {esc }}, \eta_{c, k, j}=\gamma_{c} / \gamma_{k, j}\left(\right.$ but $=1$ when $\left.\gamma_{c} \geq \gamma_{k, j}\right), \gamma_{c}=E_{c} /[(1-\beta \mu) 2.8 k T]$, and $\gamma_{k, j}=$ $\left(\epsilon_{0} / 2\right) \kappa_{\mathrm{SR}}^{k-1} \kappa_{\mathrm{ICS}}^{j-1} \eta_{\|}$. For millisecond pulsars, a slightly different formula is adopted (see Zhang \& Harding 1999). When calculating the X-ray luminosity within a certain band, $E_{c 1}<E<E_{c 2}$, we then have $L_{\Delta E}=L_{x, n t h}\left(E_{c 2}\right)-L_{x, n t h}\left(E_{c 1}\right)$. 


\section{RESULTS AND DISCUSSIONS}

The observation versus model prediction of the $\gamma$-ray and X-ray pulsars are shown in Fig.1 and Fig.2. For the X-ray luminosities, three components (the non-thermal, the full surface thermal and the hot polar cap thermal components) are taken into account.

An obvious conclusion is that the full polar cap cascade model within the framework of Harding \& Muslimov acceleration model can both reproduce the $L_{\gamma} \propto\left(L_{\mathrm{sd}}\right)^{1 / 2}$ and $L_{x}(R O S A T) \sim 10^{-3} L_{\mathrm{sd}}$ feature simultaneusly. The former was not done by the outer gap model which interprets non-thermal X-ray emission as the SR of the downward cascade from the outer gap (Cheng \& Zhang 1999; Zhang \& Cheng 1997). In our model, we also compare the non-thermal X-ray luminosity with the luminosities of the two thermal components. It is found that for middleaged pulsars such as Vela, Geminga, PSR 1055-52, and PSR 0656+14, the full surface thermal luminosity is comparable to the non-thermal one, so that such a component should be detectable from these pulsars. Such a feature is actually observed. For the hot polar cap thermal component, our model shows that it is detectable in relative old pulsars such as PSR 0950+08 and PSR 1929+10, although the non-thermal component is also detectable. The outer gap model actually only predicts pure thermal components in these pulsars, since the thick outer gap does not exist in their magnetospheres (Cheng \& Zhang 1999). For the millisecond pulsars, our model predicts that the thermal emission from polar cap heating is the dominant component in the "pulsed" ROSAT-band spectra, while the outer gap model predicts non-thermal emission, though they assume strong multipole magnetic fields near the surfaces of the millisecond pulsars. Future observations and spectral analyses can distinguish the riveling models.

\section{REFERENCES}

Arons, J., \& Scharlemann, E.T. 1979, ApJ, 231, 854

Becker, W., \& Trümper, J. 1997, A\&A, 326, 682

Cheng, K.S., Ho, C. \& Ruderman, M.A. 1986, ApJ, 300, 500

Cheng, K.S., \& Zhang, L. 1999, ApJ, 515, 337

Daugherty, J.K., \& Harding, A.K. 1996, ApJ, 458, 278

Dermer, C.D. 1990, ApJ, 360, 214

Harding, A.K., \& Muslimov, A.G. 1998, ApJ, 508, 328

Lu, T., Wei, D.M., \& Song, L.M. 1994, A\&A, 290, 815

Sturner, S.J., Dermer, C.D., \& Michel, F.C. 1995, ApJ, 445, 736

Thompson, D.J., Harding, A.K., Hermsen, W., \& Ulmer, M.P. 1997, in C.D. Dermer, M.S. Strickman, \& J.D. Kurfess (eds.), Proc. Fourth Compton Symposium, AIP Conf. Proc. 410, 39

Wei, D.M., Song, L.M., \& Lu, T. 1997, A\&A, 323, 98

Zhang, B., \& Harding, A.K. 1999, ApJ, submitted

Zhang, L. \& Cheng, K.S. 1997, ApJ, 487, 370 